\title{
Emergence of Enterobacteriaceae co-producing CTX-M-15, ArmA and PMQR in Poland
}

\author{
Katarzyna Piekarska ${ }^{A, D, F}$, Katarzyna Zacharczuk, Tomasz Wołkowicz' , Natalia Wolaniuk', \\ Magdalena Rzeczkowska ${ }^{B}$, Rafał Gierczyński ${ }^{\mathrm{E} F}$ \\ Department of Bacteriology, National Institute of Public Health-National Institute of Hygiene, Warszawa, Poland \\ A - research concept and design; $\mathrm{B}$ - collection and/or assembly of data; $\mathrm{C}$ - data analysis and interpretation; \\ $D$ - writing the article; $E$ - critical revision of the article; $F$ - final approval of the article
}

Address for correspondence

Katarzyna Piekarska

E-mail: kpiekarska@pzh.gov.pl

\section{Funding sources}

This research was partially supported with a grant No. NN 404085040 from the National Science Centre (NCN) and with an internal grant from National Institute of Public Health-National Institute of Hygiene (NIZP-PZH) No. 4/EM/2017.

Conflict of interest

None declared

Received on April 26, 2017

Reviewed on July 9, 2017

Accepted on August 9, 2018

Published online on November 30, 2018

Cite as

Piekarska K, Zacharczuk K, Wołkowicz T, et al. Emergence of Enterobacteriaceae co-producing (TX-M-15, ArmA and PMQR in Poland. Adv Clin Exp Med. 2019;28(2):249-254. doi:10.17219/acem/94165

DOI

10.17219/acem/94165

Copyright

Copyright by Author(s)

This is an article distributed under the terms of the

Creative Commons Attribution Non-Commercial License

(http://creativecommons.org/licenses/by-nc-nd/4.0/)

\begin{abstract}
Background. Plasmid-mediated extended-spectrum $\beta$-lactamases (ESBLs), 16S rRNA methylases and quinolone resistance mechanisms (PMQRs) are well-known agents conferring resistance to more than 1 antimicrobial in its group. The accumulation of these agents poses, therefore, a serious risk to public health.

Objectives. The objective of this study was to investigate the presence of common B-lactamases and 165 rRNA methylases in Qnr-producing Enterobacteriaceae and their genetic relatedness.

Material and methods. We examined 18 Qnr-producing isolates (Klebsiella pneumoniae $n=8$, Enterobacter cloacae $n=6$ and Escherichia coli $n=4$ ) selected from a collection of 215 ciprofloxacin-resistant strains obtained from patients in a 1030-bed tertiary hospital from 1 March to 31 August 2010. The antibiotics minimum inhibitory concentration (MIC) was determined using E-test. The detection of common B-lactamases, 165 rRNA methyltransferases and PMQR genes was performed using polymerase chain reaction (PCR) and sequencing. Genetic relatedness was assessed with pulsed-field gel electrophoresis (PFGE) and multilocus sequence typing (MLST).

Results. All the isolates tested were susceptible to carbapenems and colistin, while 16 were multidrug-resistant. Thirteen, 2 and 2 isolates carried qnrB1, qnrA1 and qnrS1, respectively. Ten of 13 qnrB1-positive Enterobacteriaceae also carried genes encoding for aac $\left(6^{\prime}\right)-1 b$-cr and at least 1 ESBL. The blacTX-M-15 gene was the most common ESBL. The most prevalent combination of genes was anrB1+aac $\left(6^{\prime}\right)-1 b-\left(r+b l a_{\text {TEM-1 }}+b l a_{\text {CTX-M-15. }}\right.$. Two isolates of $K$. pneumoniae and $E$. cloacae were found to bear multiple extended range resistance traits: ArmA, CTX-M-15, QnrB1, and AAC (6')-|b-cr. The PFGE showed that most of the isolates exhibited individual DNA patterns, whilst MLST assigned K. pneumoniae ( $\mathrm{n}=8$ ) to 5 sequence types (STS) (ST15, ST323, ST336, ST147, and ST525), E. coli $(n=4)$ to 2 (ST131 and ST1431) and E. cloacae $(n=5)$ to 4 (ST90, ST89, ST133, and the novel ST407).
\end{abstract}

Conclusions. Our findings reveal the accumulation of resistance traits and their important role in spreading of multiresistant bacteria among hospitalized patients.

Key words: PMQR, ESBLS, MDR, Enterobacteriaceae, Poland 


\section{Introduction}

Currently, the global spread of multidrug resistant (MDR) bacteria is considered a major public health concern. In the last decade, we have witnessed a dramatic increase in the number of MDR Enterobacteriaceae. Data from the European Antimicrobial Resistance Surveillance Network (EARS-Net) ${ }^{1}$ indicates that a majority of the Escherichia coli and Klebsiella pneumoniae had combined resistance to third generation cephalosporins, aminoglycosides and fluoroquinolones. Furthermore, the combined resistance has increased significantly also in Poland since 2010, especially for K. pneumoniae, with levels ranging from $23 \%$ to $52.5 \%$ in $2013 .{ }^{1}$ However, data on molecular characteristics of MDR Enterobacteriaceae simultaneously resistant to the aforementioned antimicrobial agents is still insufficient in Poland.

Because of their broad-spectrum antimicrobial activity, fluoroquinolones are commonly used agents in clinical and veterinary medicine to treat a wide variety of microbiological infections. The stepwise mutations in gyrase and topoisomerase IV subunits are recognized as the most common mechanism of quinolone resistance in Enterobacteriaceae. Moreover, plasmid-mediated quinolone resistance (PMQR) determinants were also reported in MDR strains as contributing to the increase of fluoroquinolones resistance. ${ }^{2}$ We previously ${ }^{3}$ described the coexistence of PMQR determinants ( $q n r \mathrm{~A}, q n r \mathrm{~B}, q n r \mathrm{~S}$ and $\left.a a c\left(6^{\prime}\right)-I b-c r\right)$ and mutations in $g y r A$ and parC among fluoroquinoloneresistant clinical Enterobacteriaceae. ${ }^{3}$

It is worth noting that previous studies have shown the association of PMQR-encoding genes with other antibiotic resistance genes, such as extended-spectrum $\beta$-lactamases (ESBL), including CTX-M-15 or plasmidmediated AmpC genes. ${ }^{2,4-6}$ In addition, PMQRs may also be co-transferred with plasmid-mediated $16 \mathrm{~S}$ rRNA methylase-encoding genes. ${ }^{7}$ However, data on coexistence of PMQRs with the other resistance traits in Enterobacteriaceae collected in Poland is lacking.

In the present study, we investigated the presence of ESBLs, pAmpC and 16S rRNA methylases in Qnr-producing Enterobacteriaceae from clinical sites in Poland, and determined their genetic relatedness. We described the Qnrproducing Enterobacteriaceae strain with the accumulation of plasmid-mediated, broad range resistance traits: ArmA, CTX-M-15, QnrB1, and AAC (6')-Ib-cr that, to the best of our knowledge, has not been yet reported from Poland.

\section{Material and methods}

\section{Study population}

A retrospective study was conducted of 18 Qnr-producing Enterobacteriaceae (K. pneumoniae $\mathrm{n}=8$, Enterobacter cloacae $\mathrm{n}=6$ and $E$. coli $\mathrm{n}=4$ ) found among the previously described 215 fluoroquinolone-resistant clinical isolates collected from 2017 inpatients in a 1030-bed tertiary hospital in a period from 1 March to 31 August 2010. ${ }^{3}$

\section{Phenotypic and genotypic investigation of resistance}

Minimum inhibitory concentrations (MICs) to 11 antibiotics were determined using E-test Strips (bioMerieux, Marcy-l'Etoile, France) and interpreted according to the European Committee on Antimicrobial Susceptibility Testing (EUCAST) recommendations (http://eucast.org). For the Qnr-producing isolates, amplification of genes encoding ß-lactamases of the most common families was performed with primers for $b l a_{\mathrm{TEM}}, b l a_{\mathrm{SHV}}$ and $b l a_{\text {CTX-M. }}{ }^{8}$ The multiplex polymerase chain reaction (PCR) with family-specific primers which detected the plasmidmediated AmpC genes bla $a_{\mathrm{MOX}}, b l a_{\mathrm{FOX}}, b l a_{\mathrm{EBC}}, b l a_{\mathrm{ACC}}$, $b l a_{\mathrm{DHA}}$, and $b l a_{\mathrm{CIT}}$ was performed as described previously. ${ }^{9}$ The characterization of $16 \mathrm{~S}$ rRNA methyltransferase genes $(\operatorname{arm} A, r m t A, r m t B$, and $r m t C)$ was done in isolates with resistance to amikacin with PCR using primers as described previously. ${ }^{10}$ Furthermore, the DNA sequencing of genes encoding for PMQRs, the selected B-lactamases and $16 \mathrm{~S}$ rRNA methyltransferase ArmA was performed. The nucleotide sequences of the PCR products were determined using conventional Sanger method. All the DNA sequences were compared with sequences in the GenBank database using the BLAST algorithm (http://www.blast. ncbi.nlm.nih.gov).

\section{Molecular typing}

The genetic relatedness of tested isolates was studied by pulsed-field gel electrophoresis (PFGE) and multilocus sequence typing (MLST). The PFGE was conducted using $\mathrm{XbaI}$ endonuclease. The PFGE patterns were analyzed using BioNumerics software v. 6.6 (Applied Maths, Sint-Martens-Latem, Belgium). Sequence types (STs) were determined using 7 highly conserved housekeeping genes according to appropriate MLST schemes. ${ }^{11-13}$ The MLST Databases available at http://mlst.warwick.ac.uk/mlst/dbs/ Ecolia; http://pubmlst.org/ecloacae/ and http://bigsdb.web. pasteur.fr were used for assigning each sequence type.

\section{Plasmid isolation and electrophoresis}

The plasmid DNAs from Qnr-producing isolates was extracted using the NucleoBond PC20 kit (Macherey-Nagel, Düren, Germany) according to the manufacturer's instruction. Plasmid size was determined with extraction and electrophoresis using a $0.8 \%$ agarose gel (Prona Plus, Burgos, Spain) in Tris-borate-EDTA (TBE) buffer. Klebsiella pneumoniae DM0269 containing 50 kb, 90 kb and $180 \mathrm{~kb}$, respectively, were used as molecular weight plasmid calculation. ${ }^{14}$ 


\section{Results}

\section{Multidrug resistance and genotyping of PMQR-producing isolates}

The majority of tested isolates were MDR, with resistance to antimicrobial agents showed in Table 1. Additional resistance was found to the following: amoxicillin and clavulanic acid (94\%; R >8 mg/L), cefotaxime (72\%; R >2 mg/L), ceftazidime (72\%; R > $\mathrm{mg} / \mathrm{L})$, gentamicin (67\%; R >4 mg/L), ciprofloxacin (100\%; R >1 mg/L), trimethoprim/sulfamethoxazole (78\%; R >4 mg/L), and chloramphenicol (72\%; R >8 mg/L). All isolates were susceptible to carbapenems and colistin.

Sequencing analysis showed that qnrB1, qnrA1 and qnrS1 were found in 13, 2 and 2 isolates, respectively. Ten of 13 qnrB1-positive Enterobacteriaceae also carried genes encoding for $a a c\left(6^{\prime}\right)-I b-c r$ and at least 1 ESBL (Fig. 1). Among them, the most prevalent combination was qnrB1+aac (6')$I b-c r+b l a_{\mathrm{TEM}-1}+b l a_{\mathrm{CTX}-\mathrm{M}-15}$, which was detected in 6 isolates, and the $b l a_{\mathrm{CTX}-\mathrm{M}-15}$ gene was the most common in all the QnrB1-producing isolates (61\%). Furthermore, 2 QnrB1and CTX-M-15-producing isolates (No. 088 and No. 216) possessed additionally the arm $A$ gene coding for $16 \mathrm{~S}$ rRNA methyltransferase ArmA. In contrast, pAmpC genes were not detected in any of isolates tested.

All the tested isolates were distinguished by the PFGE with similarity ranging from $50 \%$ to $92 \%$ (Fig. 1). Four clonal groups were distinguished, each encompassing strains with the PFGE profiles similarity $\geq 80 \%$. Two clonal groups (A and B) were found in K. pneumoniae and single clonal groups (C and D) occurred in E. coli and E. cloacae, respectively. By the multilocus sequence typing (MLST), 5 STs were identified for K. pneumoniae: ST15, ST323, ST336, ST147, and ST525, 2 for E. coli: ST131 and ST1431, and 4 for E. cloacae: ST90, ST89, ST133, and the novel ST407. Isolates belonging to the same PFGE clonal group belonged also to the same ST. With the single exception (clonal group $\mathrm{C}$ ), isolates belonging to the same clonal group shared the same resistance traits. In the clonal group $C$, 2 E. coli isolates (No. 155 and No. 169) of ST131 with 83\% of PFGE patterns similarity revealed different resistance traits (Fig. 1). These 2 isolates revealed multiple plasmids (5 and 6), including both large and small plasmids (Fig. 2).

\section{Discussion}

A significant increase in MDR Enterobacteriaceae has been reported worldwide. ${ }^{1}$ The occurrence of MDR is usually concomitant with the acquisition of fluoroquinolone resistance. ${ }^{2}$ According to EARS-Net data, in Poland from 2010 to 2013 fluoroquinolone resistance rates for $E$. coli remained almost constant (>25\%) but significantly increased from $33 \%$ to $70.1 \%$ for $K$. pneumoniae. ${ }^{1}$ What is noteworthy is that the horizontally transferable PMQRs, which are considered to support the development of the high-level

Table 1. Antimicrobial minimum inhibitory concentrations for the 18 Qnr-producing Enterobacteriaceae

\begin{tabular}{|c|c|c|c|c|c|c|c|c|c|c|c|c|c|}
\hline \multirow{2}{*}{ No. } & \multirow{2}{*}{ Species } & \multirow{2}{*}{ Specimen } & \multicolumn{11}{|c|}{ Minimum inhibitory concentration [mg/L] } \\
\hline & & & AMC & CTX & $\mathrm{CAZ}$ & IPM & MEM & GEN & CIP & TGC & CST & SXT & CML \\
\hline 088 & Kpn & blood & 64 & 32 & 4 & 0.190 & 0.016 & $>1024$ & 16 & 1 & 0.750 & $>32$ & 12 \\
\hline 036 & Kpn & urine & 32 & 0.190 & 0.750 & 0.125 & 0.016 & 1 & 128 & 3 & 0.750 & 2 & 64 \\
\hline 060 & Kpn & urine & 64 & 32 & 12 & 0.125 & 0.023 & 64 & 1,024 & 1.500 & 0.500 & $>32$ & $>256$ \\
\hline 158 & Kpn & urine & 16 & 48 & $>256$ & 0.125 & 0.047 & 256 & 128 & 2 & 0.380 & $>32$ & 16 \\
\hline 159 & Kpn & urine & 24 & $>256$ & 128 & 0.190 & 0.047 & 0.500 & 16 & 0.500 & 0.380 & $>32$ & 6 \\
\hline 215 & Kpn & urine & 32 & $>32$ & 256 & 0.032 & 0.125 & 64 & 128 & 0.500 & 0.500 & $>32$ & 16 \\
\hline 091 & Kpn & intubation tube swab & 24 & $>256$ & 64 & 0.250 & 0.064 & 32 & 64 & 1.500 & 0.380 & $>32$ & 6 \\
\hline 209 & Kpn & tracheotomy tube swab & 16 & 0.500 & 12 & 0.032 & 0.003 & 0.750 & 64 & 0.750 & 0.380 & 0.250 & 12 \\
\hline 003 & $\mathrm{ECl}$ & urine & $>256$ & $>256$ & 64 & 0.190 & 0.016 & 128 & 2 & 1 & 0.190 & $>32$ & $>256$ \\
\hline 092 & $\mathrm{ECl}$ & urine & 32 & $>256$ & 16 & 0.250 & 0.047 & 32 & 16 & 1.500 & 0.380 & $>32$ & $>256$ \\
\hline 110 & $\mathrm{ECl}$ & urine & $>256$ & 12 & 64 & 0.250 & 0.032 & 8 & 4 & 0.250 & 0.380 & $>32$ & $>256$ \\
\hline 118 & $\mathrm{ECl}$ & urine & $>256$ & $>256$ & $>256$ & 0.250 & 0.032 & 48 & 64 & 3 & 0.380 & $>32$ & $>256$ \\
\hline 214 & $\mathrm{ECl}$ & urine & 32 & $>256$ & 24 & 0.190 & 0.023 & 32 & 1.500 & 1 & 0.380 & $>32$ & 6 \\
\hline 216 & $\mathrm{ECl}$ & urine & $>256$ & $>256$ & $>256$ & 0.500 & 0.380 & 256 & 64 & 2 & 0.500 & $>32$ & 16 \\
\hline 169 & Ec & blood & 16 & 0.125 & 0.380 & NT & 0.016 & 12 & 64 & 0.250 & 0.500 & 0.064 & 16 \\
\hline 014 & Ec & urine & 16 & 0.047 & 0.125 & 0.190 & 0.012 & 0.750 & 64 & 0.190 & 0.380 & $>32$ & 4 \\
\hline 064 & Ec & urine & 1.50 & 0.016 & 0.064 & 0.190 & 0.016 & 0.380 & 64 & 0.190 & 0.250 & 0.047 & 2 \\
\hline 155 & Ec & urine & 24 & 32 & 8 & 0.125 & 0.016 & 0.500 & 256 & 1 & 0.500 & $>32$ & $>256$ \\
\hline
\end{tabular}

Kpn - Klebsiella pneumoniae; Ecl - Enterobacter cloacae; EC - Escherichia coli; AMC - amoxicillin-clavulanic acid; CTX - cefotaxime; CAZ - ceftazidime; IPM - imipenem; MEM - meropenem; GEN - gentamicin; CIP - ciprofloxacin; TGC - tigecycline; CST - colistin; SXT - trimethoprim/sulphamethoxazole; $\mathrm{CML}$ - chloramphenicol; NT - not tested. 


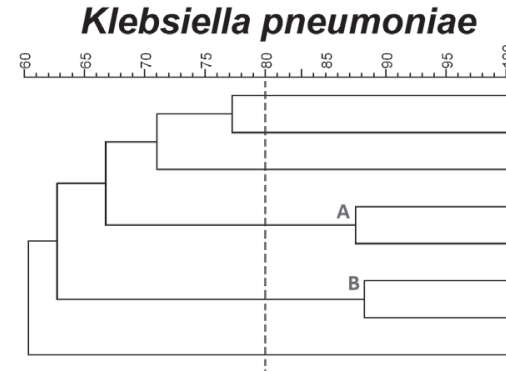

$\begin{aligned} & \text { Strain } \\ & \text { number }\end{aligned}$
060
088
159
091
215
158
209
036

ST

15

15

$$
323
$$

\section{Escherichia coli}
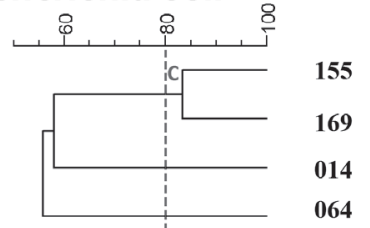

131

03.08.10

$131 \quad 28.07 .10$

$1431 \quad 05.03 .11$

- 25.05.11

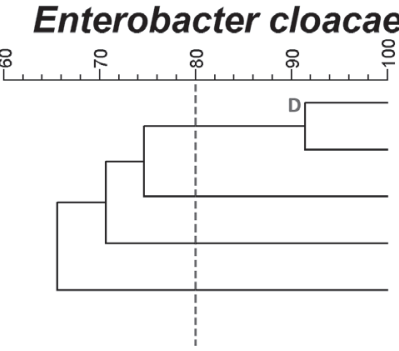

Date of

isolation

09.08.10

05.06.10

15.09.10

9.08.10

23.08.10

06.05.10
PMQR genes

profile

qnrB1, $a a c\left(6^{\prime}\right)-I b-c r$

qnrB1, $\operatorname{aac}\left(6^{\prime}\right)-I b-c r$

$q n r B 1, \operatorname{aac}\left(6^{\prime}\right)-I b-c r$

qnrB1, aac(6')-Ib-cr

qnrB1, aac(6')-Ib-cr

qnrB1

qnrB1

qnrA1 $\beta$-lactamases

profile

TEM-1, SHV-28, CTX-M-15

TEM-1, CTX-M-15

TEM-1, SHV-1, CTX-M-15 -

TEM-1, SHV-1, CTX-M-15 -

SHV-12

SHV-12

SHV-11

TEM-1, CTX-M-15

TEM-1

TEM-1

TEM-1
16S rRNA

methylase genes

qnrB1, aac(6')-Ib-cr
qnrS1
qnrS1, aac(6')-Ib-cr
qnrS1, qnrA-like

$\begin{array}{llll}003 & 90 & 04.03 .10 & q n r B 1, \operatorname{aac}\left(6^{\prime}\right)-I b-c r \\ 092 & 90 & 02.06 .10 & q n r B 1, \operatorname{aac}\left(6^{\prime}\right)-I b-c r \\ 214 & 90 & 15.09 .10 & q n r B 1, \operatorname{aac}\left(6^{\prime}\right)-I b-c r \\ 216 & 89 & 14.09 .10 & q n r B 1 \\ 118 & 407 & 23.06 .10 & q n r B 1, \operatorname{aac}\left(6^{\prime}\right)-I b-c r \\ 110 & 133 & 17.06 .10 & q n r A 1\end{array}$

TEM-1, CTX-M-15

TEM-1, CTX-M-15

TEM-1, CTX-M-15

CTX-M-15

TEM-1, CTX-M-15

SHV-12

Fig. 1. Characterization of $18 \mathrm{Qnr}$-producing Enterobacteriaceae isolates. The dendrogram based on pulsed-field gel electrophoresis (PFGE)

ST - sequence type; PMQR - plasmid-mediated quinolone resistance.

fluoroquinolone resistance, are frequently reported to spread together with ESBLs on the same plasmid. ${ }^{4-6,15}$ In this study, we screened clinical Qnr-producing Enterobacteriaceae for the presence of multiple antimicrobial resistance genes. The majority of tested isolates possessed the PMQR determinants - QnrB1 and AAC (6')-Ib-cr - and were identified mainly in combination with CTX-M-15 as the most common ESBL. However, we found no co-existence of Qnr genes and plasmid-mediated AmpC ß-lactamases determinants.

It is well-known that CTX-M-15-producing isolates are commonly multiresistant to various antibiotic agents, among them fluoroquinolones or aminoglycosides. ${ }^{4}$ Furthermore, the coexistence of QnrB1 and CTX-M-15 was also frequently observed in other European countries. ${ }^{4,5}$ Notably, our data shows that in Poland, the aforementioned combination of resistance traits was extended by the $16 \mathrm{~S}$ rRNA methyltransferase ArmA that confers the high-level resistance to almost all clinically relevant aminoglycosides. Moreover, the herein reported resistance triangle (QnrB1, CTX-M-15 and ArmA) was found in 2 Enterobacteriaceae species. Notably, one of them contained an additional PMQR gene: $a a c\left(6^{\prime}\right)-I b-c r$. Taking into account the relatively low number of isolates tested in this study, the incidence of 2 isolates with the aforementioned resistance triangle may reveal only "the tip of iceberg". Thus, our findings may argue for the need of further specific monitoring of resistance. Interestingly, the coexistence of QnrB, CTX-M-15 and ArmA in K. pneumoniae has been reported in China. ${ }^{7}$ In Europe, the coexistence of QnrA, CTX-M-15 and ArmA was reported in K. pneumoniae in Switzerland, which also coproduced CMY-16 together with carbapenemases OXA-48 and NDM-1. ${ }^{16}$ In Poland, we have also reported $K$. pneumoniae co-producing Klebsiella pneumoniae carbapenemase (KPC) with ArmA and CTX-M-3 ESBL. ${ }^{14}$ To the best of our knowledge, the presented study is the first report on clinical Enterobacteriaceae isolates co-producing PMQR, CTX-M-15 and ArmA in Poland.

In this study, we identified FQ-resistant isolates belonging to different STs possessing CTX-M-15. We found $K$. pneumoniae isolate (No. 088) and E. cloacae isolate (No. 216) to bear QnrB1, CTX-M-15 and ArmA, and belong to ST323 and ST89, respectively. Although there is not enough data to consider these 2 STs epidemic, in our study we also found K. pneumoniae of ST15, ST336, ST147, and $E$. coli of ST131. These STs have been reported in many countries and, therefore, are considered international clones. ${ }^{17,18}$ In addition, K. pneumoniae ST15 and ST336 have not been reported in Poland yet. Interestingly, in this study we found K. pneumoniae ST15 and ST336 isolates with 2 PMQRs (QnrB1 and AAC (6')-Ib-cr) and 2 ESBLs (CTX-M-15 and SHV-11 or SHV-28) accompanied with TEM-1 $\beta$-lactamase. We also observed E. coli ST131 isolate No. 155 co-producing PMQR (QnrB1 and AAC (6')-Ib-cr), TEM-1 and CTX-M-15. Interestingly, this isolate revealed 


\section{DM 0269}

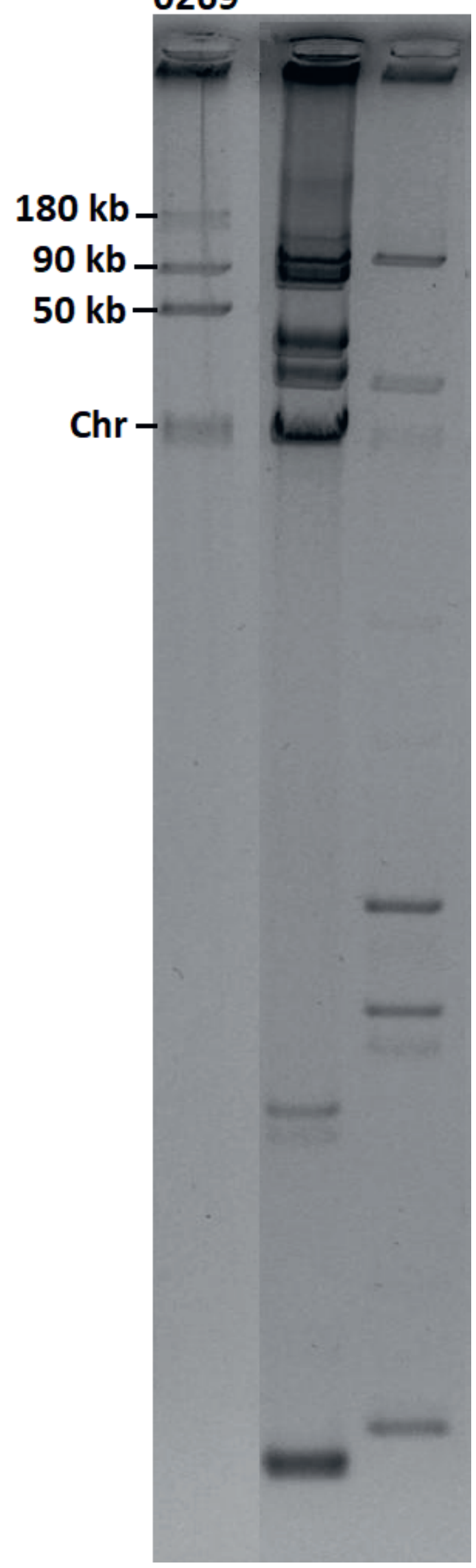

Fig. 2. Plasmids profiles
83\% PFGE-profile similarity with other ST131 isolate No. 169 that shared only QnrS1 and TEM-1. Notably, both these isolates differed by plasmid content that may reflect the dynamics of plasmid-mediated resistance traits exchange in clinical settings. Moreover, we found the novel E. cloacae ST 407 co-producing QnrB1 and AAC (6')-Ib-cr together with CTX-M-15. What is worthy of note is that all the aforementioned PMQR-producing isolates have been previously found to have mutations in quinolone resistance-determining regions (QRDR) gyrA and parC. ${ }^{3}$

In conclusion, the presented study provides baseline information on the "successful clones" possessing plasmid-mediated resistance genes providing resistance to most of clinically relevant group of antimicrobial agents including $ß$-lactams, aminoglycosides and fluoroquinolones. It is also worth noting that fluoroquinolones (mainly ciprofloxacin) are able to induce bacterial SOS response to DNA damage and potentially enhance the transmission of resistance traits by promoting horizontal dissemination of various antibiotic resistance determinants, and increase the frequency of mutants. ${ }^{19}$ The herein reported coincidence of PMQRs with other resistance traits, often multiple and broad-range plasmid-mediated mechanisms, may suggest a significant contribution of fluoroquinolone therapy in increasing the MDR phenotype in clinical Enterobacteriaceae strains.

\section{References}

1. European Centre for Disease Prevention and Control. Antimicrobial resistance surveillance in Europe 2013. Annual Report of the European Antimicrobial Resistance Surveillance Network (EARS-Net). Stockholm: ECDC, Sweden; 2014. http://ecdc.europa.eu/en/publications/ Publications/antimicrobial-resistance-europe-2013.pdf.

2. Hooper DC, Jacoby GA. Mechanisms of drug resistance: Quinolone resistance. Ann N Y Acad Sci. 2015;1354:13-31.

3. Piekarska K, Wołkowicz T, Zacharczuk K, et al. Co-existence of plasmid-mediated quinolone resistance determinants and mutations in gyrA and parC among fluoroquinolone-resistant clinical Enterobacteriaceae isolated in tertiary hospital in Warsaw, Poland. Int J Antimicrob Agents. 2015;45(3):238-243.

4. Dolejska M, Villa L, Dobiasova H, Fortini D, Feudi C, Carattoli A. Plasmid content of a clinically relevant Klebsiella pneumoniae clone from the Czech Republic producing CTX-M-15 and QnrB1. Antimicrob Agents Chemother. 2013;57(2):1073-1076.

5. Filippa N, Carricajo A, Grattard F, et al. Outbreak of multidrug-resistant Klebsiella pneumoniae carrying qnrB1 and bla $_{\mathrm{CTX}-\mathrm{M}-15}$ in a French intensive care unit. Ann Intensive Care. 2013;3:18.

6. Tóth Á, Kocsis B, Damjanova I, et al. Fitness cost associated with resistance to fluoroquinolones is diverse across clones of Klebsiella pneumoniae and may select for CTX-M-15 type extended-spectrum B-lactamase. Eur J Clin Microbiol Infect Dis. 2014;33(5):837-843.

7. Luo Y, Yang J, Zhang Y, Ye L, Wang L, Guo L. Prevalence of $\beta$-lactamases and $16 \mathrm{~S}$ rRNA methylase genes amongst clinical Klebsiella pneumoniae isolates carrying plasmid-mediated quinolone resistance determinants. Int J Antimicrob Agents. 2011;37(4):352-355.

8. Zacharczuk K, Piekarska K, Szych J, et al. Plazmid-borne 16S rRNA methylase ArmA in aminoglicoside-resistant Klebsiella pneumoniae in Poland. J Med Microbiol. 2011;60(Pt 9):1306-1311.

9. Pérez-Pérez FJ, Hanson ND. Detection of plasmid-mediated AmpC beta-lactamase genes in clinical isolates by using multiplex PCR. J Clin Microbiol. 2002;40(6):2153-2162.

10. Piekarska K, Zacharczuk K, Wołkowicz T, et al. Distribution of $16 \mathrm{~S}$ rRNA methylases among different species of aminoglycoside-resistant Enterobacteriaceae in tertiary care hospital in Poland. Adv Clin Exp Med. 2016;25(3):539-544. 
11. Miyoshi-Akiyama T, Hayakawa K, Ohmagari N, Shimojima M, Kirikae T. Multilocus Sequence Typing (MLST) for characterization of Enterobacter cloacae. PLoS One. 2013;8(6):e66358.

12. Mellmann A, Bielaszewska M, Kock R, et al. Analysis of collection of hemolytic uremic syndrome-associated enterohemorrhagic Escherichia coli. Emerg Infect Dis. 2008;14(8):1287-1290.

13. Diancourt L, Passet V, Verhoef J, Grimont PAD, Brisse S. Multilocus sequence typing of Klebsiella pneumoniae nosocomial isolates. J Clin Microbiol. 2005;43(8):4178-4182.

14. Zacharczuk K, Piekarska K, Szych J, et al. Emergence of Klebsiella pneumoniae coproducing KPC-2 and 16S rRNA methylase ArmA in Poland. Antimicrob Agents Chemother. 2011;55(1):443-446.

15. Briales A, Rodríguez-Martínez JM, Velasco C, et al. Prevalence of plasmid mediated quinolone resistance determinants qnr and $a a c\left(6^{\prime}\right)-I b$ cr in Escherichia coli and Klebsiella pneumoniae producing extendedspectrum ß-lactamases in Spain. Int J Antimicrob Agents. 2012;39(5): 431-434.
16. Seiffert SN, Marschall J, Perreten V, Carattoli A, Furrer H, Endimiani A. Emergence of Klebsiella pneumoniae co-producing NDM-1, OXA-48, CTX-M-15, CMY-16, QnrA and ArmA in Switzerland. Int J Antimicrob Agents. 2014;44(3):260-262.

17. Paltansing S, Kraakman MEM, Ras JMC, Wessels E, Bernards AT. Characterization of fluoroquinolone and cephalosporin resistance mechanisms in Enterobacteriaceae isolated in a Dutch teaching hospital reveals the presence of an Escherichia coli ST131 clone with a specific mutation in parE. J Antimicrob Chemother. 2013;68(1):40-45.

18. Rodrigues C, Machado E, Ramos H, Peixe L, Novais Â. Expansion of ESBLproducing Klebsiella pneumoniae in hospitalized patients: A successful story of international clones (ST15, ST147, ST336) and epidemic plasmids (IncR, IncFII). Int J Med Microbiol. 2014;304(8):1100-1108.

19. Beaber JW, Hochhut B, Waldor MK. SOS response promotes horizontal dissemination of antibiotic resistance genes. Nature. 2004; 427(6969):72-74. 